\title{
Deep Attentive Ranking Networks for Learning to Order Sentences
}

\author{
Pawan Kumar, ${ }^{*}$ Dhanajit Brahma, ${ }^{*}$ Harish Karnick, Piyush Rai \\ Department of Computer Science and Engineering, IIT Kanpur, India \\ \{kpawan, dhanajit, hk, piyush\}@cse.iitk.ac.in
}

\begin{abstract}
We present an attention-based ranking framework for learning to order sentences given a paragraph. Our framework is built on a bidirectional sentence encoder and a self-attention based transformer network to obtain an input order invariant representation of paragraphs. Moreover, it allows seamless training using a variety of ranking based loss functions, such as pointwise, pairwise, and listwise ranking. We apply our framework on two tasks: Sentence Ordering and Order Discrimination. Our framework outperforms various state-ofthe-art methods on these tasks on a variety of evaluation metrics. We also show that it achieves better results when using pairwise and listwise ranking losses, rather than the pointwise ranking loss, which suggests that incorporating relative positions of two or more sentences in the loss function contributes to better learning.
\end{abstract}

\section{Introduction}

Coherence is a fundamental aspect of natural language discourse and text. In a coherent discourse, normally, sentences should respect the chronological order of events. Correct logical ordering of parts of the discourse can facilitate understanding. Ordering of sentences in a discourse determines local coherence, so it is an essential aspect of natural language processing. The sentence ordering task tries to organize randomly shuffled sentences of a paragraph into a coherent text. Table 1 shows an Example of this task. (Barzilay and Lapata 2008) proposed the sentence ordering problem on the Accidents and Earthquakes datasets.

Two of the most successful recent sentence representation models, Quick Thought (Logeswaran and Lee 2018) and BERT (Bidirectional Encoder Representations from Transformers) (Devlin et al. 2018) use next sentence classification for learning the sentence representation. Next sentence classification is a special case of the sentence ordering task, which shows how vital the sentence ordering task is. Recently sentence ordering has been used in many applications like concept-to-text (Konstas and Lapata 2012), question answering (Yu et al. 2018; Verberne 2011), multi-document

\footnotetext{
${ }^{*}$ Equal contributions from both authors. Copyright (C) 2020, Association for the Advancement of Artificial Intelligence (www.aaai.org). All rights reserved.
}

\begin{tabular}{lc|cc}
\hline Unordered Paragraph & \multicolumn{2}{c}{ Ordered Paragraph } \\
\hline 3 & Then a nice thing happened. & 1 & Mario had lost his watch. \\
2 & He sent a mail to Lost \& Found. & 2 & He sent a mail to Lost \& Found. \\
4 & Somebody found his watch. & 3 & Then a nice thing happened. \\
1 & Mario had lost his watch. & 4 & Somebody found his watch. \\
\hline
\end{tabular}

Table 1: Example of unordered sentences in a paragraph (left) and ordered sentences in the same paragraph (right).

summarization (Barzilay and Elhadad 2002; Nallapati, Zhai, and Zhou 2017).

Existing state-of-the-art for sentence ordering methods, such as (Wang and Wan 2019), rely on sentence encoding with Transformer and Long Short-Term Memory (Hochreiter and Schmidhuber 1997) (LSTM) with pre-trained GloVe (Pennington, Socher, and Manning 2014) word vectors. These methods usually require sentence-by-sentence decoding to producing the reordered sentences.

We propose a novel architecture for sentence ordering and reframe the problem in a ranking framework. Our framework has several appealing properties. Firstly, while most existing works (Li and Hovy 2014; Gong et al. 2016; Chen, Qiu, and Huang 2016; Logeswaran, Lee, and Radev 2018; Cui et al. 2018; Wang and Wan 2019) use pre-trained word representations, we leverage Transformer based BERT sentence representations, allowing our model to use improved sentence encoding. The sentence encoder uses only Transformer (no LSTMs used). Following previous works we use order invariant Transformer based paragraph encoder for paragraph encoding. Secondly, while many recent works use a Pointer Network based decoder for decoding the sentence order from the encoded sentences, one sentence at a time, we propose a simple and efficient feed-forward neural network decoder. It computes a relevance score for each sentence, in parallel. These scores can be simply sorted to predict the correct sentence ordering (without expensive beam search). Thirdly, predicting scores for every sentence in this fashion allows us to reframe the sentence ordering problem as a ranking problem. Our sentence ordering model can, thus, leverage extensive prior work on the Learning to Rank framework (Burges et al. 2005).

We conduct an extensive evaluation of our model on six 
benchmark datasets for the sentence ordering task. We evaluate our model on two standard metrics: (1) Kendall's tau $(\tau),(2)$ Perfect Match Ratio (PMR). We surpass the state-ofthe-art models in terms of $\tau$ on all benchmark datasets. We also give our model's performance in terms of PMR on all the datasets. Our model excels in making accurate first and last sentence predictions, achieving better performance than previous state-of-the-art approaches. We also provide visualizations of sentence representation and sentence level attention. On the order discrimination task, we show improvements over current state-of-the-art on Accidents dataset and give competitive results on Earthquakes dataset.

\section{RankTxNet: Deep Attentive Ranking Networks for Learning to Order Sentences}

This section starts with the sentence ordering problem setup. Then we describe the proposed model RankTxNet, which as building blocks uses BERT for sentence encoding and a Transformer for paragraph encoding. We also give the details of training the model for various ranking loss functions.

\section{Problem Set-up}

The problem of sentence ordering deals with finding the correct order of sentences given a randomly ordered paragraph. In other words, the aim is to find the most coherent permutation of sentences among all possible orders in a paragraph. Given a paragraph $\mathbf{p}=\left[s_{o_{1}}, s_{o_{2}}, \cdots, s_{o_{m}}\right]$ with $m$ sentences and order $\mathbf{o}=\left[o_{1}, o_{2}, \cdots, o_{m}\right]$, paragraph $\mathbf{p}^{*}=\left[s_{O_{1}^{*}}, s_{O_{2}^{*}}, \cdots, s_{O_{m}^{*}}\right]$ is correctly ordered if $\mathbf{o}^{*}=$ $\left[o_{1}^{*}, o_{2}^{*}, \cdots, o_{m}^{*}\right]$ is the order of the most coherent permutation of sentences. For example in Table 1, the unordered paragraph is $\mathbf{p}=\left[s_{o_{1}}, s_{O_{2}}, s_{O_{3}}, s_{o_{4}}\right]=\left[s_{3}, s_{2}, s_{4}, s_{1}\right]$ and the correct order is $\mathbf{o}^{*}=[1,2,3,4]$.

\section{Model Overview and Intuition}

The proposed model has three components: a sentence encoder, a paragraph encoder and a decoder. The sentence encoder is a Transformer (Vaswani et al. 2017) based pretrained BERT (Devlin et al. 2018). The paragraph encoder is a randomly initialized Transformer network. The decoder is a simple feed forward neural network. A detailed diagram of the architecture is shown in Fig. 1.

BERT as the sentence encoder allows us to use the language modeling knowledge obtained by pre-training on freely available, large, un-annotated text data. The Transformer in the paragraph encoder ensures order invariant interaction between the input set of sentences, which is a random permutation of the desired paragraph. The order invariant representation is a result of not adding positional encoding as well as the self-attention mechanism, which attends to every sentence encoding in the set with a direct connection. While in an LSTM encoder, sentences interact through recurrent connections, which limits the flow of information between sentences occurring farther in the sequence.

FFNN decoder provides a score corresponding to each sentence in the sentence set. These scores are used for ranking the sentences to produce the correct ordering. We train our model to compute the scores for all sentences, which gives us the flexibility to use the learning to rank framework. Sorting these scores provides the correct ordering in the paragraph. A variety of ranking loss functions available in the literature can be used for studying the trade-offs between different evaluation metrics.

We mention the details of the different components in our model below.

\section{Transformer Mechanism}

We briefly describe the transformer mechanism proposed by (Vaswani et al. 2017), used in the sentence encoder as well as the paragraph encoder. Both of them use multiple self-attention layers. Each layer has a multi-head selfattention sub-layer and a position wise feed-forward sublayer. These sub-layers use residual connections (He et al. 2016), which allows easy passage of information through a deep stack of layers. Layer normalization (Lei Ba, Kiros, and Hinton 2016), LayerNorm $(x+$ Sublayer $(x))$, is also used after each sub-layer, where Sublayer $(x)$ denotes the sub-layer function.

The attention mechanism is defined on queries, keys and values packed together in matrices $\mathbf{Q}, \mathbf{K}$ and $\mathbf{V}$, respectively.

$$
\operatorname{Attention}(\mathbf{Q}, \mathbf{K}, \mathbf{V})=\operatorname{softmax}\left(\frac{\mathbf{Q K}^{\mathrm{T}}}{\sqrt{\mathrm{d}_{\mathrm{k}}}}\right) \mathbf{V}
$$

A multi-head attention for query matrix $\mathbf{Q}$, key matrix $\mathbf{K}$ and value matrix $\mathbf{V}$ is given by

$$
\begin{array}{r}
\operatorname{MultiHead}(\mathbf{Q}, \mathbf{K}, \mathbf{V})=\operatorname{Concat}\left(\mathbf{H}_{1}, \ldots, \mathbf{H}_{h}\right) \mathbf{W}^{O} \\
\text { where } \mathbf{H}_{i}=\operatorname{Attention}\left(\mathbf{Q} \mathbf{W}_{i}^{Q}, \mathbf{K} \mathbf{W}_{i}^{K}, \mathbf{V} \mathbf{W}_{i}^{V}\right)
\end{array}
$$

Here, $\mathbf{W}_{i}^{Q} \in \mathbb{R}^{d_{\text {model }} \times d_{k}}, \mathbf{W}_{i}^{K} \in \mathbb{R}^{d_{\text {model }} \times d_{k}}, \mathbf{W}_{i}^{V} \in$ $\mathbb{R}^{d_{\text {model }} \times d_{v}}$ and $\mathbf{W}^{O} \in \mathbb{R}^{h d_{v} \times d_{\text {model }}}$ are parameter matrices. Every layer in the model outputs a vector of $d_{\text {model }}$ dimensions. $d_{k}$ and $d_{v}$ are dimensions of key and value, respectively, in a single head and there are $h$ such heads in total. In self-attention, $\mathbf{Q}, \mathbf{K}$ and $\mathbf{V}$ all are from the same layer. In the sentence encoder each key, query and value is a vector corresponding to a word whereas in the paragraph encoder each such vector corresponds to a sentence.

\section{Sentence Encoder}

We use BERT for encoding each sentence in the paragraph. BERT is trained on an unsupervised Language Modeling (LM) task on English Wikipedia and BookCorpus (Zhu et al. 2015) datasets. It is jointly optimized for two LM tasks, Masked LM and Next Sentence Prediction. For Masked LM, some random words in a sentence are replaced with either a mask word [MASK] or a random word or kept unchanged. Masked LM task aims to predict the masked word correctly. Next Sentence Prediction determines whether two sentences in the input appear next to each other. In the process of learning these simple tasks on a large text corpus, BERT learns to represent sentences. Pre-trained BERT can be further finetuned for other NLP tasks. We use pre-trained BERT for sentence encoding and its parameters are also fine-tuned in an end-to-end manner for the sentence ordering task. BERT can be viewed as a multi-layer Transformer network with the layers as described in the sections above. 

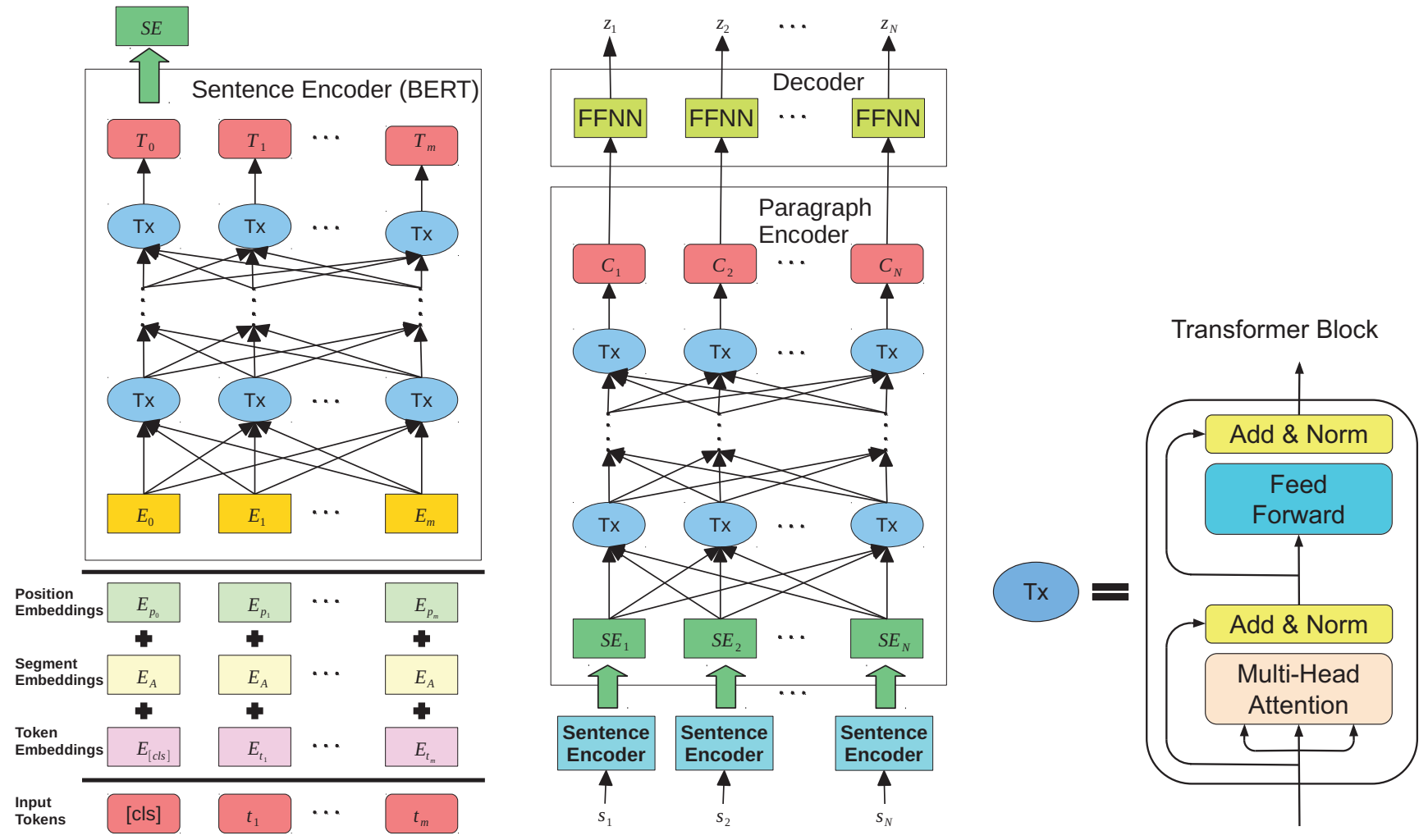

Figure 1: The proposed model architecture

\section{Paragraph Encoder}

To encode a paragraph, we again use a Transformer Network with self-attention layers. But the positional embedding layer in a standard Transformer is removed to handle the unordered nature of the sentences in the input paragraph. The paragraph encoder outputs a $d_{\text {model }}$ dimensional vector for every sentence. The encoded paragraph can be considered as a variable length vector of size $m_{n} \times d_{\text {model }}$, where $m_{n}$ is number of sentences in the $n^{t h}$ paragraph. Every node in the self-attention layer interacts with each node, including itself via length one connections. This ensures direct sharing of information among all the sentences in a paragraph.

\section{Decoder}

We use a position wise FFNN decoder that converts a sentence representation into a score, one score for each sentence. Unlike in many previous works where decoding has to be done one sentence at a time, our model computes a score for each sentence in parallel. Finally, the order of the paragraph is predicted by sorting these scores.

\section{Training and Ranking Losses}

Given a corpus with $N$ paragraphs, the $n^{t h}$ paragraph with $m_{n}$ unordered sentences is denoted by $\mathbf{p}_{n}=$ $\left[s_{1}, s_{2}, \cdots, s_{m_{n}}\right]$. We denote the score of sentence $s_{k}$ by $z_{k}$, i.e., $z_{k}=f\left(s_{k}\right)$, where $f$ is the function denoting the model. To train our model, we have used three types of loss functions. The general form of the loss function is:

$$
\text { Loss }=\frac{1}{N} \sum_{n=1}^{N} \mathcal{L}\left(\mathbf{p}_{n}\right)
$$

where $\mathcal{L}($.$) can be one of the following losses.$

Pointwise Ranking Loss: In pointwise ranking approach, we view sentence ordering as a regression problem. Each sentence in an ordered paragraph is mapped onto a realvalued gold score $y_{k} \in[0,1]$, for sentence $s_{k}$ (increasing order). For example, if a paragraph has 5 sentences then $\left[y_{1}, y_{2}, y_{3}, y_{4}, y_{5}\right]=[0,0.25,0.50,0.75,1.0]$. A similar approach for target values has been adopted by (McClure, O'Brien, and Roy 2018). The training optimizes the MSE loss:

$$
\mathcal{L}_{\text {point }}\left(\mathbf{p}_{n}\right)=\frac{1}{m_{n}} \sum_{k=1}^{m_{n}}\left(y_{k}-z_{k}\right)^{2}
$$

Pairwise Ranking Loss: For pairwise ranking approach, we take the pairwise margin ranking loss (Joachims 2002) between two consecutive sentences while training. This does not require target value as in pointwise approach. We adopt the pairwise margin ranking loss in our approach as follows:

$$
\begin{aligned}
\mathcal{L}_{\text {pair }}\left(\mathbf{p}_{n}\right) & =\frac{1}{m_{n}-1} \sum_{k=1}^{m_{n}-1} l_{p}\left(s_{k}, s_{k+1}\right) \\
l_{p}\left(s_{k}, s_{k+1}\right) & =\max \left(0, t .\left(z_{k}-z_{k+1}\right)+\gamma\right)
\end{aligned}
$$


where $t=1$ if sentence $s_{k}$ is placed in a higher position than sentence $s_{k+1}$ and $t=-1$ if sentence $s_{k+1}$ is in a higher position than $s_{k} \cdot \gamma$ is the margin hyperparameter.

Listwise Ranking Loss: The listwise ranking loss considers all the sentences in a paragraph together. We have experimented with two different listwise losses: ListNet (Cao et al. 2007) and ListMLE (Xia et al. 2008).

- ListNet: This approach (Cao et al. 2007) uses a loss based on the probability of a sentence being ranked on the top, given the scores of all the sentences in the paragraph. The top-one probability of sentence $s_{k}$ in the $n^{t h}$ paragraph using gold target values and predicted scores are denoted by $P_{n}\left(s_{k}\right)$ and $\hat{P}_{n}\left(s_{k}\right)$, respectively, which are given as:

$$
\begin{gathered}
P_{n}\left(s_{k}\right)=\frac{\exp \left(y_{k}\right)}{\sum_{i=1}^{m_{n}} \exp \left(y_{i}\right)} \\
\hat{P}_{n}\left(s_{k}\right)=\frac{\exp \left(z_{k}\right)}{\sum_{i=1}^{m_{n}} \exp \left(z_{i}\right)} \\
\mathcal{L}_{\mathrm{Net}}\left(\mathbf{p}_{n}\right)=-\sum_{k=1}^{m_{n}} P_{n}\left(s_{k}\right) \log \hat{P}_{n}\left(s_{k}\right)
\end{gathered}
$$

where $y_{k} \in[0,1]$ is the gold score for sentence $s_{k}$.

- ListMLE: In this method, the likelihood loss function is minimized, which is a surrogate loss to the perfect order based 0-1 loss function (Xia et al. 2008). Let the correct order of paragraph $n$ be $\mathbf{o}=\left[o_{1}, o_{2}, \cdots, o_{m_{n}}\right]$.

$$
\begin{array}{r}
\mathcal{L}_{\mathrm{MLE}}\left(\mathbf{p}_{n}\right)=-\log P_{M}\left(\mathbf{o} \mid \mathbf{p}_{n}\right) \\
P_{M}\left(\mathbf{o} \mid \mathbf{p}_{n}\right)=\prod_{k=1}^{m_{n}} \frac{\exp \left(z_{O_{k}}\right)}{\sum_{i=k}^{m_{n}} \exp \left(z_{o_{i}}\right)}
\end{array}
$$

Since, ListMLE gives higher scores to sentences occurring in the starting positions (contrary to our other approaches), we reverse the order to make final predictions.

\section{Related work}

Traditional Approaches: (Morris and Hirst 1991) use thesaurus for identifying lexical chains. (Lapata 2003) calculates transition probabilities between sentences and decode the sentence order greedily. (Barzilay and Lee 2004) uses topic and topic transition modeling with Hidden Markov Model (HMM). (Barzilay and Lapata 2008) extracts entities and learns entity transition probabilities. A limitation of these approaches is their reliance on linguistic domain knowledge.

Deep learning approaches: The recent trend has been towards using data-driven, end-to-end deep learning models for sentence ordering. (Chen, Qiu, and Huang 2016) predicts pairwise ordering (Pairwise Ranking Model) of sentences in the text and uses the predicted orderings in Window Network (Li and Hovy 2014). It should be noticed that our method RankTxNet with pairwise loss differs from this model in the sense that we use entire context rather than pairs of sentences, independently.

Hierarchical deep learning models have dominated recent progress in the sentence ordering task. These models have three key components: a Sentence Encoder, a Paragraph Encoder and a Decoder. The sentence encoder processes words to get sentence vectors, then the paragraph encoder uses sentence vectors for computing a paragraph or context vector. Further, these sentence and paragraph vectors are used by the decoder for computing the conditional probability of an ordering. The network is trained for maximizing conditional probability of the correct ordering.

The hierarchical recurrent neural network (RNN) model of (Gong et al. 2016; Logeswaran, Lee, and Radev 2018) has both sentence encoder and paragraph encoder based on LSTM. The decoder is an LSTM based Pointer network, which, at every time step, predicts the next sentence probability for each candidate sentence. At test time these probabilities are used in beam search for predicting the output order. The LSTM paragraph encoder processes sentences in a given order, which makes it vulnerable to the order in which sentences are provided, which is a random permutation of the correct ordering. So (Cui et al. 2018) replaced the LSTM based paragraph encoder with a Transformer (Vaswani et al. 2017) (without positional encoding) based encoder, to make it order insensitive. Recently, (Wang and Wan 2019) added a Transformer along with an LSTM in the sentence encoder to further improve the model. It is important to note that in this model, the Transformer in the sentence encoder cannot be initialized with a pre-trained BERT as it uses the output of LSTM layers rather than tokens from the input sentence. Most of the recent works use pre-trained word vectors for using linguistic knowledge from language models.

Ranking problems have been extensively studied in Learning to Rank framework (Burges et al. 2005) in many domains like information retrieval, machine translation, computational biology (Duh and Kirchhoff 2008), recommender systems (Lv et al. 2011) and software engineering (Xuan and Monperrus 2014). Properties of ranking loss functions have been well studied. They can be used for directly optimizing different properties of the sentence ordering task. We use various ranking loss functions, which are capable of utilizing the relevance score of a single sentence for the global ranking of sentences, to optimize the network.

\section{Experiments}

We conduct a comprehensive analysis of our approach on various benchmark datasets and compare our model with other state-of-the-art approaches. We also demonstrate the effectiveness of different components of our models by performing ablation analysis.

\section{Datasets}

Following (Cui et al. 2018) and previous works we run our sentence ordering experiments on NIPS abstracts, AAN/ACL abstracts and NSF abstracts datasets from (Logeswaran, Lee, and Radev 2018); arXiv abstracts and SIND/VIST captions datasets from (Gong et al. 2016; Agrawal et al. 2016; Huang et al. 2016); and ROCStory dataset from (Wang and Wan 2019; Mostafazadeh et al. 2016). Table 3 provides the statistics for each dataset. For order discrimination experiments, we use Accidents and Earthquakes datasets from (Barzilay and Lapata 2008). 


\begin{tabular}{|c|c|c|c|c|c|c|c|c|c|c|c|c|}
\hline \multirow[t]{2}{*}{ Methods } & \multicolumn{2}{|c|}{ NIPS abstracts } & \multicolumn{2}{|c|}{ AAN abstracts } & \multicolumn{2}{|c|}{ NSF abstracts } & \multicolumn{2}{|c|}{ arXiv abstracts } & \multicolumn{2}{|c|}{ SIND captions } & \multicolumn{2}{|c|}{ ROCStory } \\
\hline & $\tau$ & PMR & $\tau$ & PMR & $\tau$ & PMR & $\tau$ & PMR & $\tau$ & PMR & $\tau$ & PMR \\
\hline Entity Grid & 0.09 & - & 0.10 & - & - & - & - & - & - & - & - & - \\
\hline Seq2seq & 0.27 & - & 0.40 & - & 0.10 & - & - & - & - & - & - & - \\
\hline Window Network & 0.59 & - & 0.65 & - & 0.28 & - & - & - & - & - & - & - \\
\hline Pairwise Ranking Model & - & - & - & - & - & - & 0.66 & 33.43 & - & - & - & - \\
\hline RNN Decoder & 0.67 & - & 0.66 & - & 0.48 & - & - & - & - & - & - & - \\
\hline Variant-LSTM+PtrNet & 0.72 & - & 0.73 & - & 0.51 & - & - & - & - & - & - & - \\
\hline $\mathrm{CNN}+\mathrm{PtrNet}$ & 0.66 & - & 0.69 & - & 0.51 & - & 0.71 & 39.28 & 0.48 & 12.32 & - & - \\
\hline LSTM+PtrNet & 0.67 & - & 0.69 & - & 0.52 & - & 0.72 & 40.44 & 0.48 & 12.34 & 0.7214 & 36.25 \\
\hline ATTOrderNet & 0.72 & - & 0.73 & - & 0.55 & - & 0.73 & 42.19 & 0.49 & 14.01 & - & - \\
\hline HierarchicalATTNet & 0.6671 & 14.06 & 0.6903 & 31.29 & 0.5073 & 8.12 & 0.7536 & 44.55 & 0.5021 & 15.01 & 0.7322 & 39.62 \\
\hline RankTxNet Regression & 0.7324 & 18.91 & 0.7472 & 35.61 & 0.5607 & 9.03 & 0.7449 & 39.85 & 0.5510 & 14.03 & 0.7357 & 30.59 \\
\hline RankTxNet Pairwise & 0.7509 & 23.63 & 0.7704 & 38.86 & 0.5614 & 9.66 & 0.7516 & 41.28 & 0.5609 & 15.59 & 0.7523 & 35.30 \\
\hline RankTxNet ListNet & 0.7463 & 23.88 & 0.7644 & 37.51 & 0.5772 & 9.48 & 0.7449 & 39.26 & 0.5507 & 14.18 & 0.7483 & 33.08 \\
\hline RankTxNet ListMLE & 0.7462 & 24.13 & 0.7748 & 39.18 & 0.5798 & 9.78 & 0.7666 & 43.44 & 0.5652 & 15.48 & 0.7602 & 38.02 \\
\hline
\end{tabular}

Table 2: Kendall's tau $(\tau)$ and perfect match ratio (PMR) on test set for various benchmark datasets. Note that Kendall's tau metric, on which our method consistently outperforms other baselines, correlates with human judgements.

\begin{tabular}{lccccc}
\hline \multicolumn{1}{c}{ Datasets } & Max & Avg & \multicolumn{3}{c}{ Dataset Split } \\
\cline { 3 - 6 } & & & Train & Val & Test \\
\hline Accidents & 19 & 11.5 & 100 & - & 100 \\
Earthquakes & 32 & 10.4 & 100 & - & 99 \\
NIPS abstracts & 15 & 6 & 2448 & 409 & 402 \\
AAN abstracts & 20 & 5 & 8569 & 962 & 2626 \\
NSF abstracts & 40 & 8.9 & 96070 & 10185 & 21580 \\
arXiv abstracts & 35 & 5.38 & 884912 & 110614 & 110615 \\
SIND captions & 5 & 5 & 40155 & 4990 & 5055 \\
ROCStory & 5 & 5 & 78529 & 9816 & 9817 \\
\hline
\end{tabular}

Table 3: Train, test and validation splits along with maximum and average paragraph lengths.

\section{Hyperparameters}

For sentence encoder, we use the pre-trained BERT BASE $_{\text {B }}$ model with 12 Transformer blocks, the hidden size as 768 , and 12 self-attention heads. The feed-forward intermediate layer size is $4 \times 768$, i.e., 3072 . The paragraph encoder is a Transformer Network having 2 Transformer blocks, with hidden size 768 and a feed-forward intermediate layer size of $4 \times 768$, i.e., 3072 . We experiment with 2 , 4 and 8 Transformer blocks for ROCStory dataset; and 2 and 8 for arXiv dataset and report the best results. The 768-dimensional sentence representation obtained from Transformer is pooled by the decoder which is a five layer feed-forward network with ReLU non-linearity in each layer with hidden size of 200, and a 1-dimensional output layer for the score. We train the model with Adam optimizer (Kingma and $\mathrm{Ba} 2014$ ) with initial learning rate, $5 \times 10^{-5}$ for sentence encoder and paragraph encoder and $5 \times 10^{-3}$ for decoder; $\beta_{1}=0.9, \beta_{2}=$ 0.999 ; and batch size of 400 . For pairwise ranking loss, the value of the margin hyperparameter, $\gamma$, is set to 1 .

\section{Sentence Ordering}

Evaluation Following previous studies we use the following metrics for evaluating the sentence ordering task:

Kendall's Tau $(\tau)$ : For a sequence of length $n$, Kendall's tau $(\tau)$ is defined as $\tau=1-2 \times(\#$ inversions $) /\left(\begin{array}{l}n \\ k\end{array}\right)$. (Lapata 2006) suggests that Kendall's tau score for sentence ordering correlates with human judgements.

Perfect Match Ratio (PMR): PMR is the fraction of number of exactly correct orderings over all the paragraphs. This is the toughest evaluation metric as it does not consider any partial match. Mathematically, it can be written as PMR $=\frac{1}{N} \sum_{n=1}^{N} \mathbb{I}\left\{\hat{o}^{(n)}=o^{*(n)}\right\}$, where $\hat{o}^{(n)}$ is the predicted order and $o^{*(n)}$ is the actual correct order for $n^{t h}$ paragraph.

Baselines We compare our methods with Entity Grid (Barzilay and Lapata 2008), Seq2seq (Li and Hovy 2014), Window Network (Li and Hovy 2014), Pairwise Ranking Model (Chen, Qiu, and Huang 2016), RNN Decoder, Variant-LSTM+PtrNet (Logeswaran, Lee, and Radev 2018), CNN+PtrNet, LSTM+PtrNet (Gong et al. 2016), ATTOrderNet (Cui et al. 2018) and HierarchicalATTNet (Wang and Wan 2019). Our methods are denoted by RankTxNet Regression, RankTxNet Pairwise, RankTxNet ListNet and RankTxNet ListMLE.

Results Table 2 provides the results of the sentence ordering experiments on six benchmark datasets. Most of the results of prior approaches have been taken from (Cui et al. 2018). We consistently achieve better results than the stateof-the-art methods on $\tau$ on all the datasets. We reiterate that $\tau$ score correlates with human judgements. RankTxNet improves the previous state-of-the-art in terms of $\tau$ scores by the absolute percentage of $3.09 \%$ in NIPS abstracts, $4.48 \%$ in AAN abstracts, $2.98 \%$ in NSF abstracts, $1.3 \%$ in arXiv abstracts, $6.31 \%$ in SIND captions and $2.8 \%$ in ROCStory dataset. On SIND captions, we also get an improvement on PMR from $15.01 \%$ to $15.59 \%$. On all other datasets, we show competitive PMR score. Among our approaches, pairwise and listwise methods always outperform the pointwise method. Specifically, ListMLE performs better than all other methods in most of the cases.

SIND contains descriptions of natural images and absence of visual information makes the ordering task more difficult 


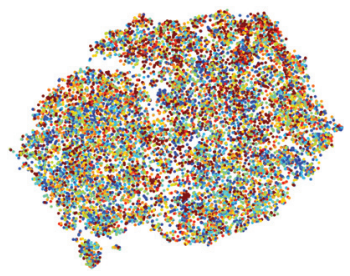

(a) AAN: Untrained

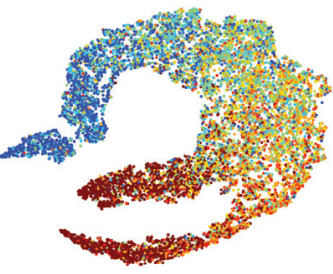

(b) AAN: Trained

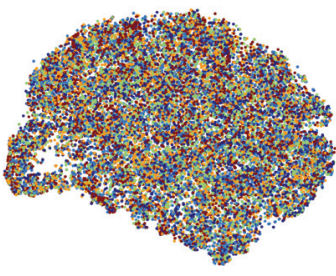

(c) SIND: Untrained

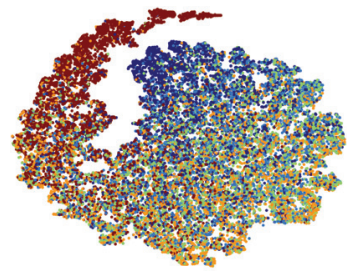

(d) SIND: Trained

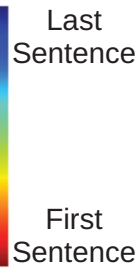

Sentence

Figure 2: t-SNE embeddings of sentence representations for AAN abstracts and SIND captions datasets on sentence ordering task. Colors correspond to position of the sentences in the original paragraph. Untrained: Sentence embeddings before finetuning (same as pre-trained BERT). Trained: Sentence embeddings after fine-tuning.

compared to other datasets. Higher maximum (40) and average (8.9) size of paragraphs make NSF a harder dataset than others, leading to lower performance.

More details about hyperparameters are provided in the supplementary material.

\begin{tabular}{lccllc}
\hline \multirow{2}{*}{ Methods } & \multicolumn{2}{c}{ SIND } & & \multicolumn{2}{c}{ arXiv } \\
\cline { 2 - 3 } \cline { 5 - 6 } & First & Last & & First & Last \\
\hline Pairwise Ranking Model & - & - & & 84.85 & 62.37 \\
CNN+PtrNet & 73.53 & 53.26 & & 89.43 & 65.36 \\
LSTM+PtrNet & 74.66 & 53.30 & & 90.47 & 66.49 \\
ATTOrderNet & 76.00 & 54.42 & & 91.00 & 68.08 \\
\hline RankTxNet Regression & 78.52 & 57.37 & & 92.59 & 68.51 \\
RankTxNet Pairwise & 79.09 & 58.69 & & 92.87 & $\mathbf{6 9 . 3 3}$ \\
RankTxNet ListMLE & $\mathbf{8 0 . 3 2}$ & $\mathbf{5 9 . 6 8}$ & & $\mathbf{9 2 . 9 7}$ & 69.13 \\
RankTxNet Listnet & 78.00 & 58.18 & & 92.46 & 68.64 \\
\hline
\end{tabular}

Table 4: Accuracy of predicting first and last sentences in SIND and arXiv datasets.

As discussed by (Gong et al. 2016; Chen, Qiu, and Huang 2016; Cui et al. 2018), it is more significant to identify the first and the last sentences in a paragraph. We provide the accuracies of our approach and compare it with the results reported in (Cui et al. 2018) in Table 4 for SIND captions and arXiv abstracts datasets. All our approaches perform better than the state-of-the-art models. In particular, on SIND dataset, our model achieves absolute improvement of $4.32 \%$ and $5.26 \%$ respectively, for first and last positions over the current state-of-the-art model.

To visualize the effect of training on the sentence representation, we use t-SNE embeddings. We show the t-SNE embeddings for AAN abstracts and SIND captions datasets before and after training in Fig. 2. It is important to note that before training, sentence representation is same as that of pre-trained BERT. The sentences are taken from the test set. Clearly, our model is learning to separate sentences in different positions and generalizes well on the unseen test set. This also shows that simply using the BERT representations is not enough as the t-SNE embeddings do not show any pattern before training the model on sentence ordering task. Figure 3 shows the visualization for paragraph encoder self-attentions.

\begin{tabular}{lcc}
\hline \multicolumn{1}{c}{ Methods } & Accidents & Earthquakes \\
\hline Graph & 84.6 & 63.5 \\
HMM+Entity & 84.2 & 91.1 \\
HMM & 82.2 & 93.8 \\
Entity Grid & 90.4 & 87.2 \\
Recurrent & 84.0 & 95.1 \\
Recursive & 86.4 & 97.6 \\
Discriminative Model & 93.0 & 99.2 \\
Variant-LSTM+PtrNet & 94.4 & 99.7 \\
LSTM+PtrNet & 93.7 & 99.5 \\
ATTOrderNet & 96.2 & $\mathbf{9 9 . 8}$ \\
\hline RankTxNet & $\mathbf{9 6 . 7 9}$ & 98.65 \\
\hline
\end{tabular}

Table 5: Performance of different models for order discrimination task on Accidents and Earthquakes datasets.

\section{Order Discrimination}

We present evaluation of our model on the Order Discrimination task defined in (Barzilay and Lapata 2008; Elsner and Charniak 2008; 2011), in this section. For a given paragraph and its randomly permuted sentences, the objective of the order discrimination task is to discriminate between the original and permuted paragraphs. We analyze the models using percentage accuracy on this binary classification task.

For order discrimination we use the best sentence ordering model, found by validation on heldout data, to predict ordering for both original and permuted paragraphs. We compute Kendall's Tau (higher if the number of inverted pairs is lower) for both predicted ordering, with respect to ordering $o=[0,1,2, \ldots, m]$, where $m$ is the number of sentences in the paragraph. The paragraph with higher Kendall's Tau value is classified as an original or more coherent paragraph.

Following (Bertolino, Marchetti, and Muccini 2005; Logeswaran, Lee, and Radev 2018; Cui et al. 2018), we evaluate our models on Accidents and Earthquakes datasets for order discrimination so that we can compare their performance with the current state-of-the-art models. We use 1986 and 1956 test pairs on Accidents and Earthquakes, respectively, with the same setup as in (Barzilay and Lapata 2008).

Results We compare our models with Graph (Guinaudeau and Strube 2013), HMM and HMM+Entity (Louis and 


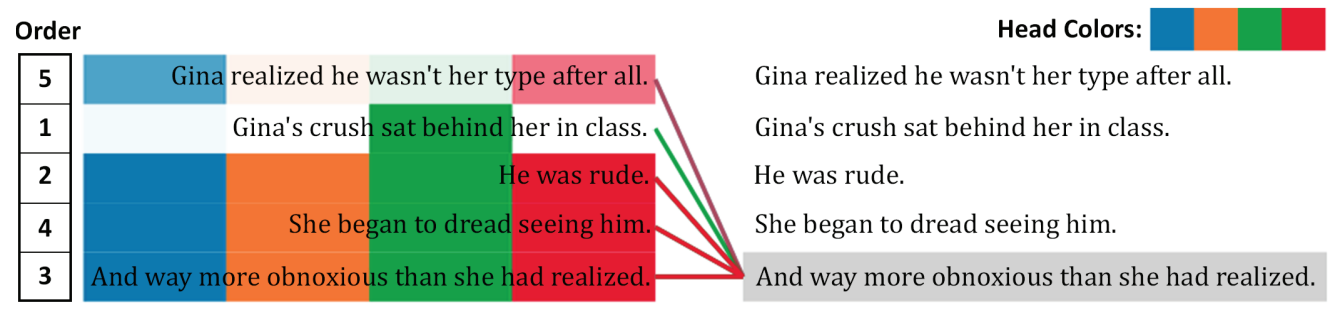

Figure 3: Self-attention scores of last layer of paragraph encoder: Attention score for all heads are shown in different colors. Higher Intensity represent higher values. First and last sentences can be differentiated, clearly. Sentences with positive and negative sentiments have different patterns in attention values.

Nenkova 2012), Entity Grid (Barzilay and Lapata 2008), Recurrent and Recursive (Li and Hovy 2014), Discriminative model (Li and Jurafsky 2016), Variant-LSTM+PtrNet (Logeswaran, Lee, and Radev 2018), CNN+PtrNet and LSTM+PtrNet (Gong et al. 2016) and ATTOrderNet (Cui et al. 2018). Order discrimination results are reported in Table 5 .

On Accidents, we outperform the current state-of-the-art and on Earthquakes, our model gives competitive results. We report our best performing method ListMLE on this task.

\section{Ablation Analysis}

In this section, we conduct various ablation studies to assess our model and understand the roles played by different components in our model.

Finetune vs. No Finetune BERT: To analyze how BERT is contributing as a sentence encoder without further training on the sentence ordering dataset, we make the parameters of BERT sentence encoder non-trainable. We conduct these experiments on AAN abstracts and SIND captions for all of our approaches. Fig. 4 shows that not fine-tuning BERT on the sentence ordering dataset reduces the performance drastically. This suggests that fine-tuning BERT/sentence encoder in an end-to-end fashion for this particular task contributes to significantly better learning.

Pretrained vs. Random Transformer: To observe the effect of using BERT pre-trained model for sentence encoder, we run experiments on AAN abstracts and SIND captions using a randomly initialized Transformer and BERT initialized Transformer (our model). All weights in both the models are fine-tuned for the sentence ordering task. Fig. 4 shows that the performance reduces substantially for random Transformer. This indicates that our model is able to utilize the language modeling knowledge from pre-trained BERT for sentence ordering.

We observe similar ablations results for NIPS and ROC.

\section{Conclusion and Future work}

We propose a novel ranking loss and Transformer based RankTxNet for sentence ordering and order discrimination task. RankTxNet uses pre-trained BERT for sentence representation and a Transformer for paragraph representation. It uses a simpler feed forward network for decoding, compared to previous work. The model can be trained on extensively studied ranking loss functions, opening a new direction for

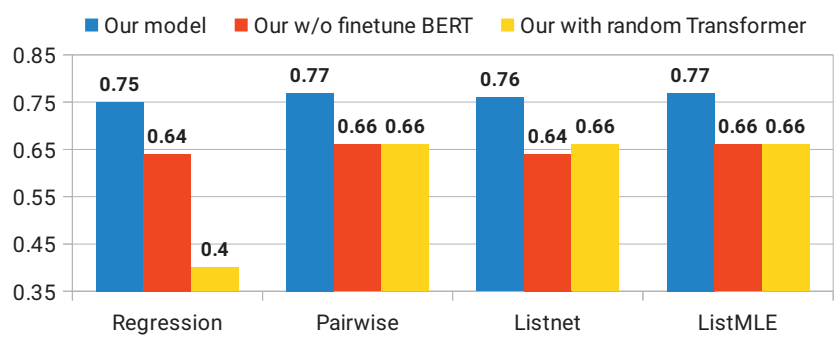

(a) AAN abstracts

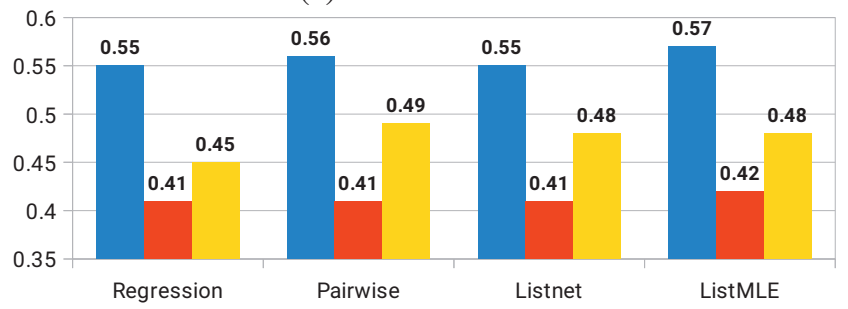

(b) SIND captions

Figure 4: Comparison of Kendall's tau $(\tau)$ for our different models on AAN abstracts and SIND captions datasets with variants: i. Without fine-tuning BERT/sentence encoder, ii. Using randomly initialized BERT/sentence encoder.

exploiting advances in the ranking literature. Our experiments demonstrate ability of the model to learn sentence ordering. RankTxNet has improved the state-of-the-art results in sentence ordering and order discrimination tasks on various benchmark datasets. We report Kendall's tau and perfect match ratio on six datasets for the sentence ordering task. The simple feed forward network in the decoder can be easily replaced by any complex network. In a learning to rank setting different metrics have been optimized, directly. Future work on sentence ordering can explore these methods. Our model can be extended for other ordering problems.

Acknowledgements: The authors thank the anonymous reviewers for their feedback. PR acknowledges support from the Visvesvaraya Young Faculty Fellowship by MeitY, India. PK is supported by the Research-I Foundation at IIT Kanpur.

\section{References}

Agrawal, H.; Chandrasekaran, A.; Batra, D.; Parikh, D.; and Bansal, M. 2016. Sort story: Sorting jumbled images and 
captions into stories. arXiv preprint arXiv:1606.07493.

Barzilay, R., and Elhadad, N. 2002. Inferring strategies for sentence ordering in multidocument news summarization. Journal of Artificial Intelligence Research.

Barzilay, R., and Lapata, M. 2008. Modeling local coherence: An entity-based approach. Computational Linguistics.

Barzilay, R., and Lee, L. 2004. Catching the drift: Probabilistic content models, with applications to generation and summarization. In HLT-NAACL. ACL.

Bertolino, A.; Marchetti, E.; and Muccini, H. 2005. Introducing a reasonably complete and coherent approach for model-based testing. ENTCS.

Burges, C.; Shaked, T.; Renshaw, E.; Lazier, A.; Deeds, M.; Hamilton, N.; and Hullender, G. N. 2005. Learning to rank using gradient descent. In ICML.

Cao, Z.; Qin, T.; Liu, T.-Y.; Tsai, M.-F.; and Li, H. 2007. Learning to rank: from pairwise approach to listwise approach. In ICML. ACM.

Chen, X.; Qiu, X.; and Huang, X. 2016. Neural sentence ordering. arXiv preprint arXiv:1607.06952.

Cui, B.; Li, Y.; Chen, M.; and Zhang, Z. 2018. Deep attentive sentence ordering network. In EMNLP. ACL.

Devlin, J.; Chang, M.-W.; Lee, K.; and Toutanova, K. 2018. Bert: Pre-training of deep bidirectional transformers for language understanding. arXiv preprint arXiv: 1810.04805.

Duh, K., and Kirchhoff, K. 2008. Learning to rank with partially-labeled data. In SIGIR. ACM.

Elsner, M., and Charniak, E. 2008. Coreference-inspired coherence modeling. In ACL-HLT. ACL.

Elsner, M., and Charniak, E. 2011. Extending the entity grid with entity-specific features. In ACL-HLT.

Gong, J.; Chen, X.; Qiu, X.; and Huang, X. 2016. End-toend neural sentence ordering using pointer network. arXiv preprint arXiv:1611.04953.

Guinaudeau, C., and Strube, M. 2013. Graph-based local coherence modeling. In $A C L$.

He, K.; Zhang, X.; Ren, S.; and Sun, J. 2016. Deep residual learning for image recognition. In $C V P R$.

Hochreiter, S., and Schmidhuber, J. 1997. Long short-term memory. Neural Comput.

Huang, T.-H. K.; Ferraro, F.; Mostafazadeh, N.; Misra, I.; Agrawal, A.; Devlin, J.; Girshick, R.; He, X.; Kohli, P.; Batra, D.; et al. 2016. Visual storytelling. In HLT-NAACL.

Joachims, T. 2002. Optimizing search engines using clickthrough data. In ACM SIGKDD. ACM.

Kingma, D. P., and Ba, J. 2014. Adam: A method for stochastic optimization. arXiv preprint arXiv:1412.6980.

Konstas, I., and Lapata, M. 2012. Concept-to-text generation via discriminative reranking. In $A C L$. ACL.

Lapata, M. 2003. Probabilistic text structuring: Experiments with sentence ordering. In ACL. ACL.

Lapata, M. 2006. Automatic evaluation of information ordering: Kendall's tau. Computational Linguistics.
Lei Ba, J.; Kiros, J. R.; and Hinton, G. E. 2016. Layer normalization. arXiv preprint arXiv:1607.06450.

Li, J., and Hovy, E. 2014. A model of coherence based on distributed sentence representation. In EMNLP.

Li, J., and Jurafsky, D. 2016. Neural net models for open-domain discourse coherence. arXiv preprint arXiv:1606.01545.

Logeswaran, L., and Lee, H. 2018. An efficient framework for learning sentence representations. arXiv preprint arXiv:1803.02893.

Logeswaran, L.; Lee, H.; and Radev, D. 2018. Sentence ordering and coherence modeling using recurrent neural networks. In $A A A I$.

Louis, A., and Nenkova, A. 2012. A coherence model based on syntactic patterns. In EMNLP. ACL.

Lv, Y.; Moon, T.; Kolari, P.; Zheng, Z.; Wang, X.; and Chang, Y. 2011. Learning to model relatedness for news recommendation. In $W W W$.

McClure, D.; O'Brien, S.; and Roy, D. 2018. Context is key: New approaches to neural coherence modeling. arXiv preprint arXiv:1812.04722.

Morris, J., and Hirst, G. 1991. Lexical cohesion computed by thesaural relations as an indicator of the structure of text. Comput. Linguist.

Mostafazadeh, N.; Chambers, N.; He, X.; Parikh, D.; Batra, D.; Vanderwende, L.; Kohli, P.; and Allen, J. 2016. A corpus and evaluation framework for deeper understanding of commonsense stories. arXiv preprint arXiv:1604.01696.

Nallapati, R.; Zhai, F.; and Zhou, B. 2017. Summarunner: A recurrent neural network based sequence model for extractive summarization of documents. In $A A A I$.

Pennington, J.; Socher, R.; and Manning, C. 2014. Glove: Global vectors for word representation. In EMNLP.

Vaswani, A.; Shazeer, N.; Parmar, N.; Uszkoreit, J.; Jones, L.; Gomez, A. N.; Kaiser, Ł.; and Polosukhin, I. 2017. Attention is all you need. In NIPS.

Verberne, S. 2011. Retrieval-based question answering for machine reading evaluation. In CLEF.

Wang, T., and Wan, X. 2019. Hierarchical attention networks for sentence ordering. In $A A A I$.

Xia, F.; Liu, T.-Y.; Wang, J.; Zhang, W.; and Li, H. 2008. Listwise approach to learning to rank: theory and algorithm. In $I C M L$. ACM.

Xuan, J., and Monperrus, M. 2014. Learning to combine multiple ranking metrics for fault localization. In ICSME.

Yu, A. W.; Dohan, D.; Luong, M.-T.; Zhao, R.; Chen, K.; Norouzi, M.; and Le, Q. V. 2018. Qanet: Combining local convolution with global self-attention for reading comprehension. arXiv preprint arXiv:1804.09541.

Zhu, Y.; Kiros, R.; Zemel, R.; Salakhutdinov, R.; Urtasun, R.; Torralba, A.; and Fidler, S. 2015. Aligning books and movies: Towards story-like visual explanations by watching movies and reading books. In CVPR. 